\title{
Development of an Aberration Corrected 1.2-MV Field Emission Transmission Electron Microscope
}

Hiroyuki Shinada $^{1}$, Toshiaki Tanigaki ${ }^{1}$, Tetsuya Akashi ${ }^{1}$, Yoshio Takahashi ${ }^{1}$, Tadao Furutsu ${ }^{1}$, Tomokazu Shimakura ${ }^{1}$, Takeshi Kawasaki ${ }^{1}$, Keigo Kasuya ${ }^{1}$, Heiko Müller ${ }^{2}$, Maximilian Haider ${ }^{2}$, Nobuyuki Osakabe ${ }^{1}$, and Akira Tonomura ${ }^{1,3}$

${ }^{1}$ Central Research Laboratory, Hitachi, Ltd., Hatoyama 350-0395, Japan

${ }^{2}$ Corrected Electron Optical Systems GmbH, Englerstr. 28, D-69126, Heidelberg, Germany

${ }^{3}$ RIKEN Center for Emergent Matter Science (CEMS), Wako 351-0198, Japan

A practical holography electron microscope was first developed in 1978 by the late Dr. Tonomura ${ }^{[1]}$. After that, we (Tonomura's group) developed bright and monochromatic field-emission electron beams over 35 years for observing quantum phenomena by utilizing the wave nature of electrons. As it turns out, every time we developed a brighter electron beam, electron interference experiments became easier to perform, and the precision in the phase measurements increased, thereby opening up new application fields.

Atomic-resolution electromagnetic field analysis of non-periodic local structures such as interfaces is important in developing heterostructures and bulk materials with boundaries because their properties derive from local electromagnetic characteristics. For example, the development of rare-earth permanent magnets with high coercive force at high temperatures is required for the efficient motors used in hybrid or electric vehicles. Improvements in their coercive force are expected by controlling their grain boundary structures and their magnetic properties ${ }^{[2]}$. Thus, electron microscopes need to be developed for observing electromagnetic fields at atomic resolution.

For this purpose, a 1.2-MV cold field-emission transmission electron microscope (TEM) equipped with a spherical-aberration corrector ${ }^{[3]}$ has been developed (Fig. 1). The microscope has the following superior properties: stabilized accelerating voltage (stability: $0.3 \mathrm{ppm}$ peak to peak), minimized electrical and mechanical fluctuation, and a field emission gun with high stability and brightness ${ }^{[4]}$. Information transfer of 43 pm was accomplished by using $\mathrm{W}\{633\}$ chromatic lattice fringes. When this developed FE-TEM was applied to observations of GaN [411] thin samples, the projected Ga atom positions were visualized with $44 \mathrm{pm}$ separation-the smallest separation ever observed (Fig. 2). This resolution is an important base performance for effective electron holography observations. The microscope enables performing electromagnetic field observations at high-resolution that were not possible with 300-kV TEMs in various types of research. 
References:

[1] A. Tonomura et al., Jpn. J. Appl. Phys. 18, 9 (1979).

[2] H. Sepehri-Amin et al., Acta Mater. 61, 6622 (2013).

[3] M. Haider et al., Nature 392, 768 (1998).

[4] K. Kasuya et al., J. Vac. Sci. Technol. B 32, 031802 (2014).

[5] This research was supported by a grant from the Japan Society for the Promotion of Science (JSPS) through the Funding Program for World-Leading Innovative R\&D on Science and Technology (FIRST Program),” initiated by the Council for Science and Technology Policy (CSTP).

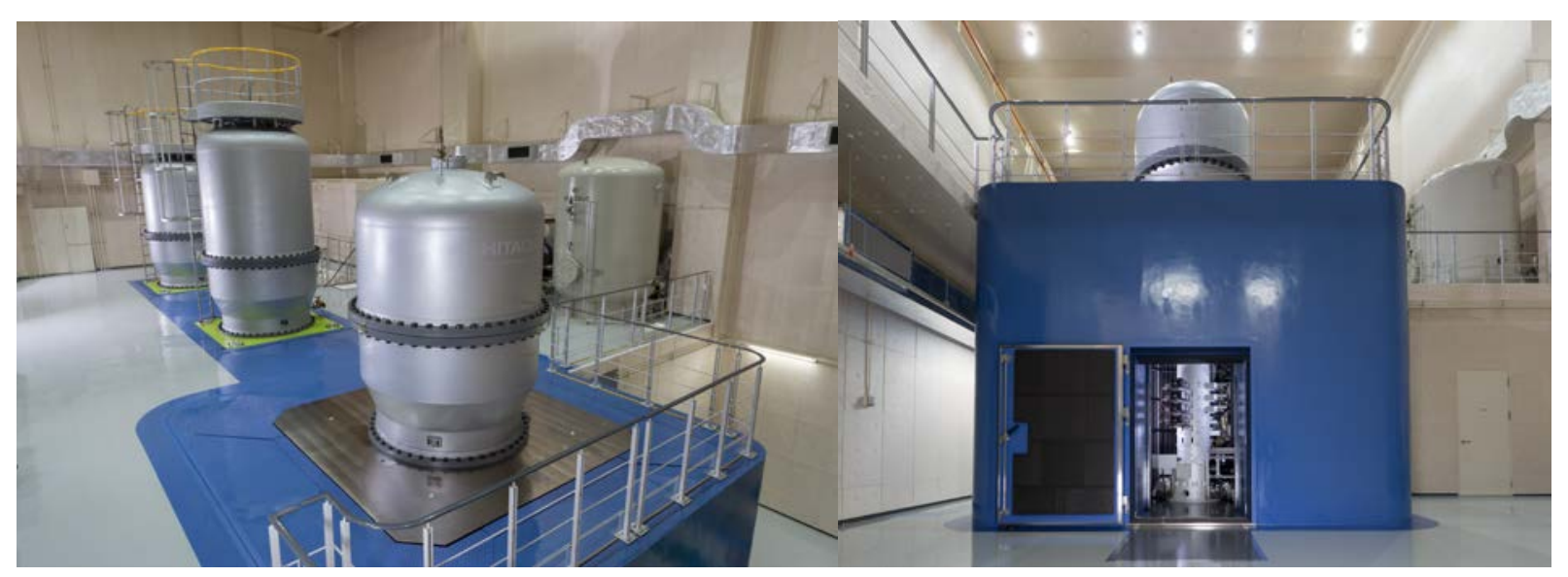

Figure 1. Aberration corrected 1.2-MV cold field-emission transmission electron microscope.
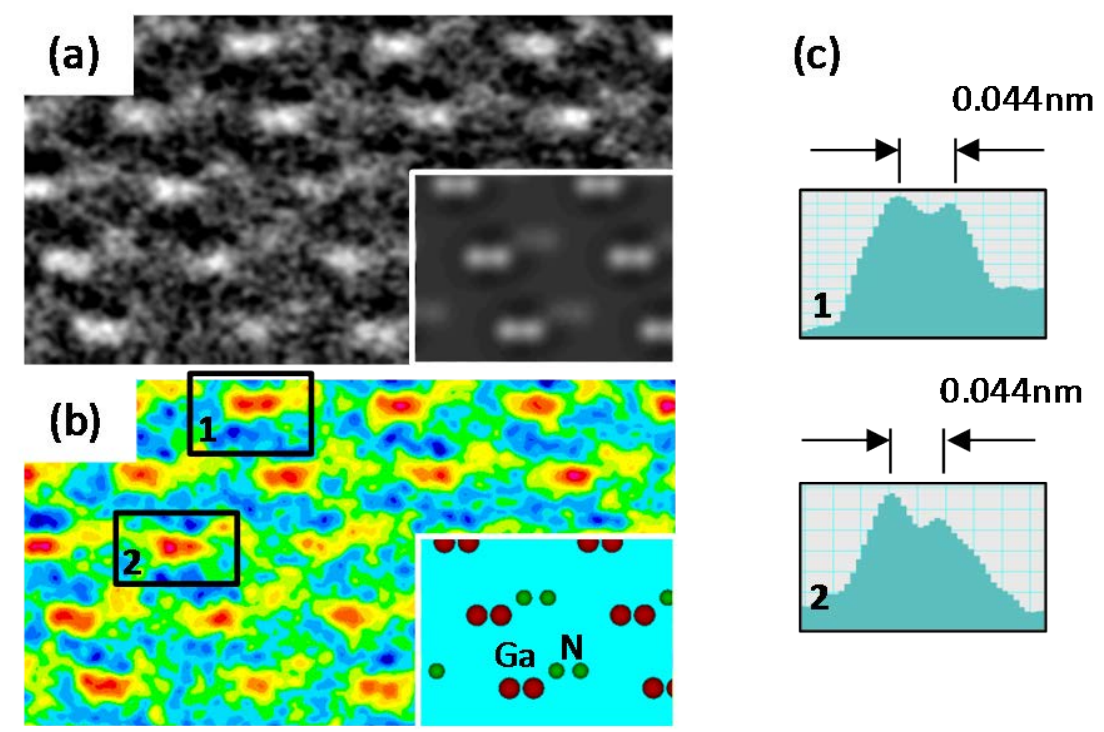

Figure 2. (a) High-resolution TEM image of GaN [411] thin sample. Projected Ga atom positions (white arrows) with 44-pm separation were clearly observed. Inset shows corresponding simulated image. (b) Corresponding Gaussian low-pass filtered image. (c) Line profiles of Ga atom pairs indicated by black rectangles 1 and 2 in (b). 